\title{
Understanding the CD28/CTLA-4 (CD152) Pathway and Its Implications for Costimulatory Blockade
}

\author{
D. Gardner ${ }^{1}$, L. E. Jeffery ${ }^{1}$ and D. M. Sansom ${ }^{2, *}$ \\ ${ }^{1}$ University of Birmingham, MRC Centre for Immune \\ Regulation, Birmingham, UK \\ ${ }^{2}$ UCL Institute of Immunity and Transplantation, Royal \\ Free Campus, London, UK \\ *Corresponding author: David M. Sansom, \\ d.sansom@ucl.ac.uk
}

T cell activation is a key event in the adaptive immune system and vital in the generation of protective cellular and humoral immunity. Activation is required to generate CD4 effector $T$ cell responses and provide help for $B$ cell and cytotoxic $T$ cell responses. While defective $T$ responses to foreign antigen result in infectious pathology, over-reactive $T$ cell responses against self-antigens result in autoimmunity and, in a transplantation setting, tissue rejection. Understanding how $T$ cell activation is normally regulated is critical to therapeutic intervention and the CD28/CTLA-4 (CD152) pathway represents the initial activation checkpoint in molecular terms. In particular, while the CTLA-4 pathway is well established as an essential regulator of self-reactivity, its mechanism of action is still uncertain. Such mechanistic issues are important given its central position in $T$ cell activation and the increasing number of therapeutic modalities aimed at manipulating the CD28/CTLA-4 pathway. Here, we provide an updated view of CTLA-4 biology, reviewing the established features of the system and highlighting its interplay with CD28. We then discuss how recent progress in our understanding of this pathway affects our interpretations following intervention.

Abbreviations: dABs, domain antibodies; Tregs, regulatory $\mathrm{T}$ cells

Received 08 April 2014, revised 19 May 2014 and accepted for publication 20 May 2014

\section{A Functional Overview of the CD28/CTLA-4 Pathway}

CD28 is expressed on the surface of the majority of naïve CD4 and CD8 T cells and is the major costimulatory molecule in initial T cell activation. Together with engagement of the T cell receptor CD28 ligation results in the augmentation of many aspects of $T$ cell-mediated immunity (1-3). Conse- quently, mice deficient in CD28 show an array of immune defects including impaired T cell activation, a lack of $T$ cell help for $\mathrm{B}$ cells and poor memory $\mathrm{T}$ cell responses, all highlighting the importance of CD28 costimulation in the generation of effective $\mathrm{T}$ cell responses and immune memory.

The immune stimulatory features of the CD28 pathway are triggered by engagement of two well-described ligands found on antigen-presenting cells (4). The two ligands CD80 (B7/BB1 or B7-1) and CD86 (B7-2) were, until recently, thought to be the sole ligands for CD28 and CTLA-4; however, there are recent reports that human (but not mouse) CD28 and CTLA-4, can also bind to the ICOS ligand (5). In addition, the PD-1 ligand, PD-L1 can also interact with CD80 (6). The significance of these novel interactions is still emerging and will not be discussed further here. Importantly, the expression of CD80 and CD86 is up-regulated in response to inflammatory stimuli including Toll-like receptor stimulation. As such, up-regulation of ligands is seen as a key link between innate "danger" signals and the triggering of an effective adaptive immune response (7). Despite structural and affinity differences (8) which would suggest functional differences, to date, the current view is that CD80 and CD86 have largely redundant or overlapping functions as represented in Figure $1(9,10)$.

In addition to binding to CD28, both CD80 and CD86 also bind to the inhibitory protein CTLA-4, which is a CD28 homologue, expressed on activated $T$ cells and especially regulatory $T$ cells (Tregs) (11). From a functional perspective, the most striking observation is that in contrast to CD28, where deficiency leads to lack of effective $T$ cell responses, CTLA-4-deficient mice suffer from a fatal overactivated phenotype resulting in profound autoimmunity driven by self-reactive $T$ cells $(12,13)$. This functional dichotomy between CD28 and CTLA-4 is even more striking given that these two diametrically opposite outcomes are controlled by interactions with the same ligands. The CD28/CTLA-4 pathway is therefore perhaps best viewed as an integrated system, which controls the balance between $\mathrm{T}$ cell activation and self-tolerance, in a process that is influenced by the expression of two shared ligands.

\section{Fundamental Concepts}

At present, there are a large number of interpretations of how the CTLA-4 pathway functions based on a wide range 


\section{Gardner et al}

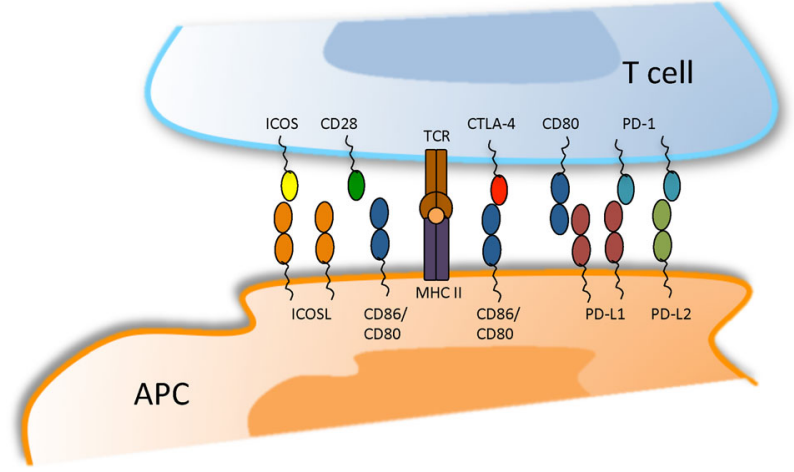

Figure 1: Shared interactions between CD28 and CTLA-4 family members. Two main ligands, CD80 and CD86 (drawn generically here for clarity), are present on activated antigenpresenting cells (APC) and can bind to both CD28 and CTLA-4. Ligand binding to $C D 28$ provides costimulatory signals in addition to TCR stimulation whereas CTLA-4 acts to inhibit ligand-driven CD28 function. Additional interactions between CD80 and the PD-1 ligand PD-L1 and interactions between the ICOS ligand and human CD28/CTLA-4 have also been proposed. The sharing of ligands between activatory and inhibitory receptors means the pathway is an integrated system and as such perturbations in one interaction can influence others in the pathway.

of experiments. We will not try to cover all experimental observations, but concentrate on selected ideas in order to generate a conceptual framework for understanding how the various manipulations of the CD28/CTLA-4 pathway available clinically might impact immune function.

\section{CTLA-4 as an inhibitory signal?}

Based predominantly on studies using agonistic anti-CTLA-4 antibodies, ideas relating to CTLA-4 function have frequently focused on the concept of an inhibitory signal preventing $T$ cell activation $(14,15)$. According to this concept, ligand binding to CTLA-4 (up-regulated on activated T cells) would be expected to generate intrinsic inhibitory signals that "switch off" T cell activation, proliferation and IL-2 production. This has been expanded to include roles for the direct recruitment of phosphatases (16), effects on T cell adhesion and motility (17) and proposed roles for various splice variants of CTLA-4 (18-20) with presumed inhibitory signaling capacity. The concept that emerges from these studies is that CTLA-4 possesses an inhibitory signaling capacity. However, there are still questions over the extent to which cross-linked antibodies to CTLA-4 are a faithful mimic of CTLA-4 engagement by its ligands and the nature of inhibitory signals still remains uncertain. Therefore, while numerous cell-intrinsic concepts for CTLA-4 function have been proposed and discussed (21-24) the role of such signals in CTLA-4 function in vivo remains unestablished.

\section{A cell extrinsic function for CTLA-4 in vivo}

A major challenge to the physiological importance of the cell-intrinsic signaling mechanisms mentioned above is a series of straightforward and widely repeated experiments in chimeric mice that possess both CTLA-4 WT and CTLA-4deficient $T$ cells. This reveals that mice containing mixtures of CTLA-4-/- and CTLA-4+/+ T cells fail to develop lethal lymphoproliferative disease and the CTLA-4-/- T cells maintain a normal nonactivated phenotype (25-27). It follows that the critical CTLA-4 functions, required to prevent systemic autoimmunity, are therefore $T$ cellextrinsic. Such data fit well with the possibility of CTLA-4 acting in a suppressive manner, such as an effector molecule on Tregs, but are much less compatible with direct inhibitory signaling in effector T cells. In support of the concept of extrinsic regulation it has become increasingly evident that CTLA-4 is indeed an important component of Treg function. This issue has been convincingly demonstrated by recent experiments using Tregs from CTLA-4-deficient mice (28) or conditionally deficient Tregs (29), which reveal CTLA-4 to be critical for Treg function in the prevention of autoimmunity. Further evidence for the importance of CTLA-4 to Tregs has recently come from the demonstration that expression of CTLA-4 and repression of IL-2 expression are two essential components to confer a Treg phenotype (30).

\section{CD28 and CTLA-4 functions are tightly connected}

The sharing of ligands between CD28 and CTLA-4 highlights the key principle that CD28 and CTLA-4 functions are closely intertwined. Importantly, it is evident that the fatal phenotype caused by CTLA-4 deficiency occurs as a result of CD28-dependent and ligand-driven $T$ cell activation. Accordingly, CTLA-4-deficient mice lacking both ligands, those with CD28 deficiency or those treated with ligand blocking compounds (e.g. CTLA-4-lg) all have substantially ameliorated disease $(31,32)$. Thus, the concept that emerges is that a key role of CTLA-4 is to directly control stimulation of the CD28 pathway via its natural ligands.

\section{Transendocytosis as a mechanism for CTLA-4 function}

Interestingly, the principle of using CTLA-4 to inhibit CD28 function by preventing access to ligands underpins the development of both abatacept and belatacept, yet has traditionally not been widely thought of as a mechanism for CTLA-4 function physiologically. However, we recently identified a novel molecular mechanism of CTLA-4 function, which exploits exactly this principle. Based initially on observations using cell coculture models we observed that robust transfer of ligands (CD80 and CD86) could occur between ligand-expressing donor cells in contact with CTLA-4 expressing recipient cells (33). This observation prompted the idea that CTLA-4 could potentially act as a physical ligand-capturing device thereby depleting shared CD28-ligands from antigen-presenting cells. Subsequent experiments revealed that the entire ligand (either CD80 or CD86) including its cytoplasmic domain could be transferred to the CTLA-4 recipient cell and that internalized ligands were subsequently degraded consistent with the 
known pattern of CTLA-4 intracellular trafficking (34). This process occurs in vivo and is seen only in CD4+ CD25+ T cells (including, but not restricted to Tregs) and removal of ligands by transendocytosis can therefore be considered as a cell-extrinsic form of ligand blockade. This process is reminiscent of the mechanism used by abatacept or belatacept, with the difference being that with transendocytosis ligand is physically removed as opposed to being simply blocked.

Consistent with the concept of transendocytosis, considerable evidence indicates that CTLA-4 is able to alter the levels of CD80 and CD86 on antigen-presenting cells $(29,35-37)$. Transendocytosis by CTLA-4 therefore provides a plausable explanation for how this can be achieved as well as a cogent reason why CD28 and CTLA-4 share ligands: If this were not the case then CTLA-4 would be unable to control CD28 stimulation in this model. In terms of functional significance, the reduction in the level of costimulation available through CD28 may be sufficient to suppress activation of weakly self-reactive $T$ cells that emerge from the thymus and thus CTLA-4 expression can regulate autoimmunity. Taken together transendocytosis provides an alternative and simple model of antigenspecific, $T$ cell-extrinsic suppression compatible with a function for CTLA-4 on Tregs and which encompasses many of the known features of CTLA-4 biology.

\section{Manipulation of the CD28/CTLA-4 pathway}

The above discussion highlights the fact that CD28 engagement represents a key checkpoint in the activation of T cells and that CTLA-4 may directly oppose this function. Therefore, rather than viewing CD28 and CTLA-4 as receptors that function separately, the pathway is best viewed as an integrated (and finely balanced) system with CTLA-4 providing a counter balance to CD28 function. Perturbation of this balance can therefore have a number of potential consequences depending on the functional mechanisms that are considered to be at play. Below we consider several different manipulation approaches and how they may potentially affect immune function in both obvious and less obvious ways.

\section{Ligand Blockade}

Ligand blockade has to date been the most widely exploited approach for manipulation of the CD28/CTLA-4 pathway. Blocking antibodies to CD80 and CD86 have been developed as a strategy for blocking the CD28/CTLA-4 pathway; however, despite some potential advantages for tailoring immune responses, selective ligand blockade has not been widely applied in a clinical setting.

In contrast to selective blockade of CD80 and CD86, simultaneous blockade of both ligands has been widely exploited in the context of the fusion protein CTLA-4-Ig now available clinically in two forms: abatacept and belatacept
(Figure 2A and B). By exploiting the higher affinity of CTLA-4 for its ligands, these compounds have been shown to inhibit $T$ cell responses in models of autoimmune disease and in transplantation models $(38,39)$.

The humanized version of CTLA-4-Ig, which incorporates a modified IgG Fc domain (abatacept), has been approved for the treatment of moderate to severe rheumatoid arthritis in patients who have not responded to other diseasemodifying drugs (40). However, due to the limited efficacy of abatacept in nonhuman primate models of transplantation, attention has now turned to an artificially engineered higher-affinity CTLA-4-Ig variant, LEA29Y (belatacept). This sequence modification translates into elevated affinity for both CD86 and CD80 with an associated increased potency of CD28 inhibition $(41,42)$. Belatacept also has an altered bias in that it has a greater relative increase in CD86 binding compared to CD80. Belatacept has now been approved for clinical use in immunosuppression regimens following renal transplantation $(43,44)$.

While blocking CD28 ligands with abatacept and belatacept is simple in concept, there are a number of theoretical considerations affecting the use of these compounds. Rather obviously, the first is that such approaches can realistically only hope to inhibit responses that are in fact CD28 dependent. What makes a T cell response CD28 dependent is not entirely clear but is generally thought to involve the intensity of TCR engagement, such that strong TCR stimuli may require less CD28 costimulation (45). A second issue is that memory $T$ cells may have lower requirements for $\mathrm{CD} 28$ and may therefore be more difficult to control via costimulation blockade. Together these caveats may relate to the difficulty in translating effective blockade in animal models, which are predominantly immunologically naive into the human setting where alloresponses may come from established memory clones as well as naïve $T$ cells.

Another consideration relating to ligand blockade is its potential impact upon Treg biology. The generation, function and maintenance of Tregs appear to be CD28dependent processes (46-48). Furthermore, the generation of induced Tregs from naive T cells may also involve CD28 signals (49), although this issue is contoversial (50). Despite potential loss of Tregs due to ligand blockade, this is not normally problematic since effector responses are concomitantly inhibited. Thus, the relative impact on Tregs versus the degree of control over effectors may be a significant factor in outcome. Interestingly, in one study, in an $\mathrm{MHC}$ II-mismatched cardiac transplantation model, CTLA-4-Ig promoted allograft rejection in a manner that was associated with a loss of natural Tregs and changes in the effector T cell:Treg ratio. In contrast, in a different transplant model the dominant impact of CTLA-4-Ig was the suppression of effector $\mathrm{T}$ cell responses with significantly enhanced allograft survival (51). In line with this issue, treatment with belatacept has been associated with higher incidences of 


\section{Gardner et al}
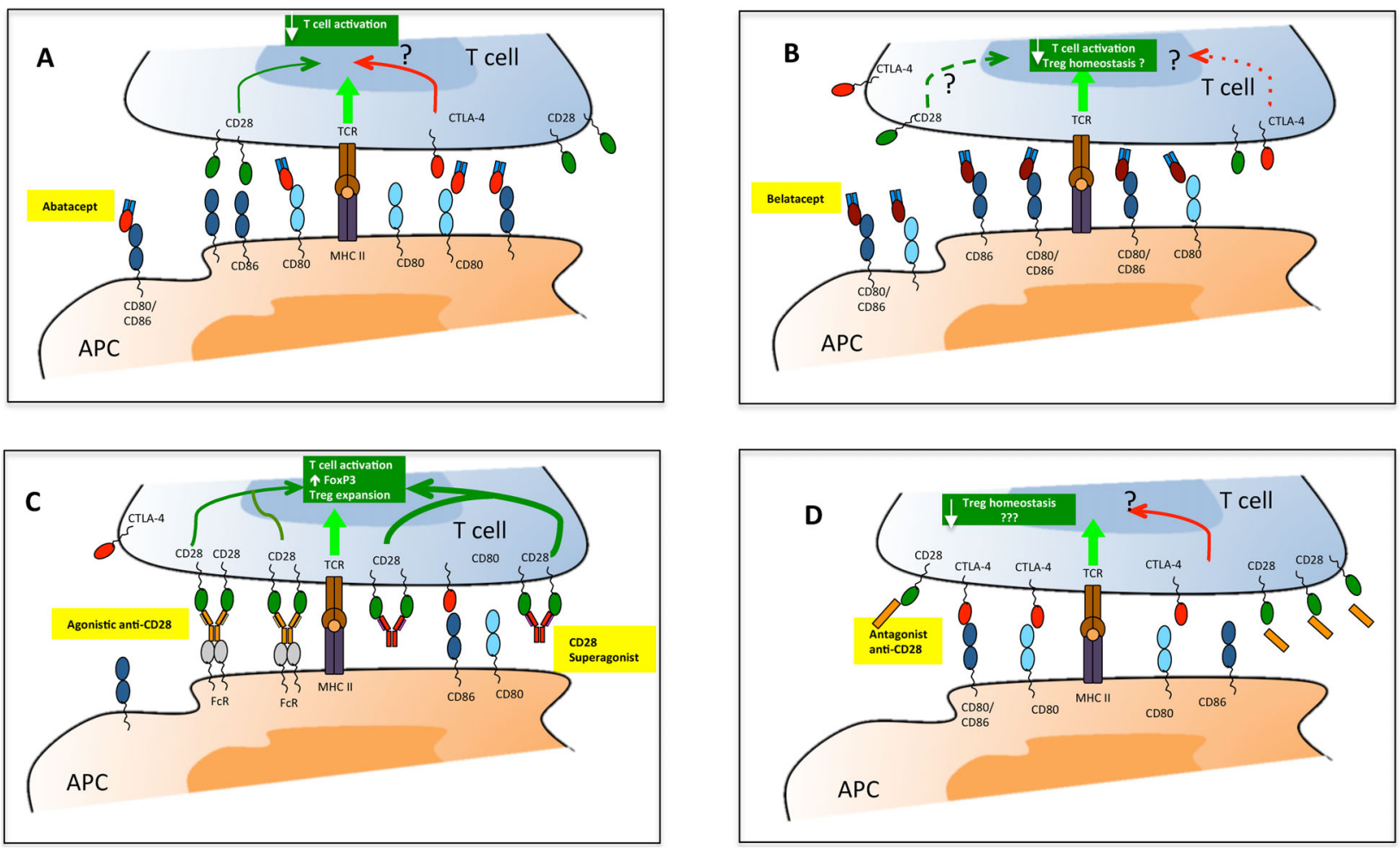

Figure 2: Possible outcomes of manipulating CD28/CTLA-4 pathway. Four possible manipulation strategies (A-D) are shown with the modifying agent highlighted in yellow. In (A) treatment with abatacept binds to both CD86 (dark blue) and CD80 (light blue). Abatacept has the same natural ligand binding affinities as CTLA-4 and as such has a strong preference for CD80 occupancy. This translates into incomplete occupancy of CD86 with more complete binding to CD80. Such an approach diminishes but does not ablate CD28 signaling and may leave sufficient CD86 available to promote regulatory T cell (Treg) survival while still attenuating CD28-dependent T cell activation. (B) Belatacept has an engineered higher affinity for both CD80 and CD86. This results in more potent blockade of both ligands and increased capacity to inhibit T cell responses. However, there is a possibility that such potent inhibition of CD28 could impair the homeostasis of Tregs, which are thought to be CD28 dependent. Targeting CD28 directly can exhibit agonistic (C) or antagonistic effects (D). Agonists can promote T cell activation but also may have the possibility to selectively expand Tregs at appropriate doses. The potential for generating unwanted $T$ cell stimulation is increased with this approach. (D) CD28 antagonists are the most direct approach to blocking costimulation. However, these antibodies need to be rigorously screened for unwanted agonistic activity. High-affinity antagonists should prevent $T$ cell activation while leaving CTLA-4 ligands available. However, CD28 blockade may also have unwanted effects on Treg homeostasis. In all the scenarios above, the impact on CTLA-4 function (red) is dependent on the molecular mechanism by which CTLA-4 is deemed to work (see main text). The red arrow denotes CTLA-4 function and not an inhibitory signal per se. The impact of inhibiting the above pathways on CTLA-4 function remains the biggest uncertainty.

acute rejection (52) especially at higher doses (53), which may be due to more effective CD28 blockade suggesting possible effects on Tregs (Figure 2B).

Another potential issue of ligand blockade is in relation to putative CTLA-4 inhibitory signals. If intrinsic CTLA-4 signaling is important for the control of normal effector $T$ cell responses, then blocking the ligands that generate such inhibition is potentially deleterious (Figure 2B). In such a case, exacerbated immune responses might be expected. There appear to be examples of enhanced responses due to both abatacept treatment (51) and belatacept treatment, associated with an increased prevalence of acute rejection (52). However, the mechanisms underlying any increase in responses following ligand blockade are presently unclear. It will therefore be important to discern if such enhanced immune responsiveness relates to an inhibitory effect on Tregs or an intrinsic enhancement of conventional T cell responses by removing CTLA-4 inhibition. Possible effects on Tregs include decreased levels of IL-2 available due to effective inhibition of production by conventional $\mathrm{T}$ cells or alternatively blocking intrinsic CD28 signals required for Treg homeostasis. The fact that problems are less apparent with abatacept compared to belatacept may suggest that weaker blockade by abatacept may have some Treg sparing effect.

\section{Anti-CD28 Specific Antibodies}

Given the potential for undesirable effects of ligand blockade strategies, CD28 blockade has been explored as 
an alternative in transplantation tolerance and autoimmune treatment. However, this approach has its own challenges since anti-CD28 antibodies can have agonistic as well as antagonistic effects (Figure $2 \mathrm{C}$ and $\mathrm{D}$ ), leading to polyclonal $T$ cell expansion rather than stimulation blockade. Thus for CD28 blockade to be viable, the possibility of agonistic activity has to be carefully considered. Importantly, antibodies that display agonistic effects in vitro have often proven antagonistic in vivo, inhibiting disease and reducing rejection of heart, liver and skin allografts (reviewed in (54)). Whether agonistic or antagonistic properties are evident may therefore depend on context, for example, in the presence of an effective ligand-driven CD28 signaling antiCD28 antibodies are often antagonistic, whereas in the absence of ligands agonistic properties can be observed.

Perhaps counterintuitively, superagonistic anti-CD28 antibodies that potently stimulate $T$ cell activation (55) have been observed to restore immune tolerance in several autoimmune and transplantation models (56-58). This presents something of a conundrum as to whether enhancing Treg (superagonists) or blocking effector cells (antagonists) is the better strategy. The former strategy of using CD28 costimulation to expand Treg (Figure 2C) was initially behind the ill-fated TGN1412 trial (59), suggesting it is extremely problematic. However, recent studies have continued to explore this approach and indicate that selective Treg expansion is nonetheless possible with such an approach (60).

The opposite approach to generate more efficient and specifically antagonistic CD28 blocking drugs continues to be explored (Figure 2D). Genetic fusion of the long-lived serum protein $\alpha 1$-anti-trypsin with the single-chain-Fv fragment from anti-CD28 (sc28AT) successfully increased the antibody half-life and reduced proliferation of human and primate $T$ cells in mixed lymphocyte reactions and inhibited cytokine production (61). More recently there has been a report of increases in the frequency of functional FoxP3+ and CTLA-4+ Tregs in the blood associated with CD28 blockade (62). This finding is surprising given the evidence that CD28 signals are generally pro-Treg generation and survival. If confirmed this suggests that a balance can been achieved between CD28 blockade and loss of Tregs. It will then be of interest to determine if such Tregs are thymically derived or induced in this setting. The authors have continued to develop further CD28 blocking reagents and have shown one of these (FR104) prevents graftversus-host disease in a humanized mouse model in a CTLA-4-dependent manner (63). Similar data were also obtained using a CD28Fv in a cardiac allograft model (64). Thus, it seems that while CD28 blockade affects Treg homeostasis, their (in vitro) suppressive function can still be maintained (62).

Other anti-CD28 fragment approaches have also been tried using single domain antibodies (dABs) or nanobodies. These comprise only a single $\mathrm{VH}$ domain and are therefore small enough to be easily cloned in bacteria and selected for high antigen affinity through phage or ribosome display. Recently, the development of two dABs was reported that were $>10$-fold more potent for binding to CD28 than either sc28AT or FR104 and more effectively inhibited human mixed lymphocyte responses (65). One attraction behind all the CD28 antibody approaches is the potential for preserved CTLA-4 function. For example, CD28 blockade does not prevent downregulation of CD86 on dendritic cells by CTLA-4+ T cells (65). However, this point may be, to some extent, moot depending on our understanding of CTLA-4 biology. If CTLA-4 acts by competition for, or removal of, CD28-ligands then effective CD28 blockade renders the continued availability of ligands largely redundant. If, however, CTLA-4 acts by receiving ligand generated signals then CD28 blockade will continue to allow additional ligand-driven inhibitory function. In this respect, recent reports suggest that anti-CD28 dAb allowed CTLA-4dependent induction of the co-inhibitory protein 2B4, whose expression on CD8+ T cells might be important for enhancing graft survival (66). Importantly, in this study, Liu et al (66) also showed that their anti-CD28 dAb could substantially increase skin allograft survival. Again the nature of the CTLA-4 involvement in this model remains to be fully defined. The authors' interpretation is that CD28 blockade allows the transmission of additional inhibitory signals via CTLA-4, which are then revealed using antiCTLA-4 blockade. As highlighted above this concept is not consistent with much of the basic literature on CTLA-4 in vivo (22). An alternative interpretation is that anti-CTLA-4 treatment directly affects Treg function, which works alongside the blockade of effector responses via CD28. Thus, while CD28 blockade would appear to be a very fruitful strategy for immune modulation, precise details in understanding mechanism of action are awaited.

The last two decades have witnessed very substantial progress in manipulating the immune response via the CD28/CTLA-4 pathway for therapeutic benefit. This progress has moved somewhat ahead of our fundamental understanding of how the pathway operates. The mechanistic gaps in our understanding, particularly relating to CTLA-4, now need to be filled in order that precise strategies for controlling effector $T$ cells while preserving natural Treg functions can be fully optimized.

\section{Disclosure}

The authors of this manuscript have no conflicts of interest to disclose as described by the American Journal of Transplantation.

\section{References}

1. Linsley $P$, Ledbetter $J$. The role of the CD28 receptor during $T$ cell responses to antigen. Annu Rev Immunol 1993; 11: 191-212. 


\section{Gardner et al}

2. Sansom DM. CD28, CTLA-4 and their ligands: Who does what and to whom? Immunology 2000; 101: 169-177.

3. Keir ME, Sharpe AH. The B7/CD28 costimulatory family in autoimmunity. Immunol Rev 2005; 204: 128-143.

4. Sansom DM, Manzotti CN, Zheng Y. What's the difference between CD80 and CD86? Trends Immunol 2003; 24: 313-318.

5. Yao S, Zhu Y, Zhu G, et al. B7-h2 is a costimulatory ligand for CD28 in human. Immunity 2011; 34: 729-740.

6. Butte MJ, Keir ME, Phamduy TB, Sharpe AH, Freeman GJ. Programmed death-1 ligand 1 interacts specifically with the B7-1 costimulatory molecule to inhibit T cell responses. Immunity 2007; 27: 111-122.

7. Joffre O, Nolte MA, Sporri R, Reis e Sousa C. Inflammatory signals in dendritic cell activation and the induction of adaptive immunity. Immunol Rev 2009; 227: 234-247.

8. Collins A, Brodie D, Gilbert R, et al. The interaction properties of costimulatory molecules revisited. Immunity 2002; 17: 201-210.

9. Lanier L, O'Fallon S, Somoza C, et al. CD80(B7) and CD86(B70) provide similar costimulatory signals for $\mathrm{T}$ cell proliferation, cytokine production and generation of CTL. J Immunol 1995; 154: 97-105.

10. Borriello F, Sethna MP, Boyd SD, et al. B7-1 and B7-2 have overlapping, critical roles in immunoglobulin class switching and germinal center formation. Immunity 1997; 6: 303-313.

11. Walker LS. Treg and CTLA-4: Two intertwining pathways to immune tolerance. J Autoimmun 2013; 45: 49-57.

12. Tivol EA, Borriello F, Schweitzer AN, Lynch WP, Bluestone JA, Sharpe AH. Loss of CTLA-4 leads to massive lymphoproliferation and fatal multiorgan tissue destruction, revealing a critical negative regulatory role of CTLA-4. Immunity 1995; 3: 541-547.

13. Ise $W$, Kohyama M, Nutsch KM, et al. CTLA-4 suppresses the pathogenicity of self antigen-specific $T$ cells by cell-intrinsic and cell-extrinsic mechanisms. Nat Immunol 2010; 11: 129-135.

14. Krummel MF, Allison JP. CTLA-4 engagement inhibits IL-2 accumulation and cell cycle progression upon activation of resting T cells. J Exp Med 1996; 183: 2533-2540.

15. Walunas TL, Bakker CY, Bluestone JA. CTLA-4 ligation blocks CD28-dependent T cell activation. J Exp Med 1996; 183: 25412550.

16. Lee KM, Chuang E, Griffin M, et al. Molecular basis of $T$ cell inactivation by CTLA-4. Science 1998; 282: 2263-2266.

17. Schneider H, Downey J, Smith A, et al. Reversal of the TCR stop signal by CTLA-4. Science 2006; 313: 1972-1975.

18. Stumpf M, Zhou X, Bluestone JA. The B7-independent isoform of CTLA-4 functions to regulate autoimmune diabetes. J Immunol 2013; 190: 961-969.

19. Araki M, Chung D, Liu S, et al. Genetic evidence that the differential expression of the ligand-independent isoform of CTLA-4 is the molecular basis of the Idd5.1 type 1 diabetes region in nonobese diabetic mice. J Immunol 2009; 183: 5146-5157.

20. Vijayakrishnan L, Slavik JM, Illes Z, et al. An autoimmune diseaseassociated CTLA-4 splice variant lacking the B7 binding domain signals negatively in T cells. Immunity 2004; 20: 563-575.

21. Wing K, Yamaguchi T, Sakaguchi S. Cell-autonomous and nonautonomous roles of CTLA-4 in immune regulation. Trends Immunol 2011; 32: 428-433.

22. Walker LS, Sansom DM. The emerging role of CTLA4 as a cellextrinsic regulator of T cell responses. Nat Rev Immunol 2011; 11: 852-863.

23. Rudd CE. The reverse stop-signal model for CTLA4 function. Nat Rev Immunol 2008; 8: 153-160.

24. Bour-Jordan $\mathrm{H}$, Esensten JH, Martinez-Llordella M, Penaranda C, Stumpf $\mathrm{M}$, Bluestone JA. Intrinsic and extrinsic control of peripheral T-cell tolerance by costimulatory molecules of the CD28/B7 family. Immunol Rev 2011; 241: 180-205.

25. Bachmann MF, Kohler G, Ecabert B, Mak TW, Kopf M. Cutting edge: Lymphoproliferative disease in the absence of CTLA-4 is not T cell autonomous. J Immunol 1999; 163: 1128-1131.

26. Homann D, Dummer W, Wolfe T, et al. Lack of intrinsic CTLA-4 expression has minimal effect on regulation of antiviral T-cell immunity. J Virol 2006; 80: 270-280.

27. Friedline $\mathrm{RH}$, Brown DS, Nguyen $\mathrm{H}$, et al. CD4+ regulatory T cells require CTLA-4 for the maintenance of systemic tolerance. J Exp Med 2009; 206: 421-434.

28. Schmidt EM, Wang CJ, Ryan GA, et al. Ctla-4 controls regulatory $\mathrm{T}$ cell peripheral homeostasis and is required for suppression of pancreatic islet autoimmunity. J Immunol 2009; 182: 274-282.

29. Wing K, Onishi $Y$, Prieto-Martin P, et al. CTLA-4 control over Foxp3+ regulatory T cell function. Science 2008; 322: 271-275.

30. Yamaguchi $T$, Kishi $A$, Osaki $M$, et al. Construction of selfrecognizing regulatory $T$ cells from conventional $T$ cells by controlling CTLA-4 and IL-2 expression. Proc Natl Acad Sci U S A 2013; 110: E2116-E2125.

31. Tai $X$, Van Laethem $F$, Sharpe AH, Singer A. Induction of autoimmune disease in CTLA-4-/- mice depends on a specific $\mathrm{CD} 28$ motif that is required for in vivo costimulation. Proc Natl Acad Sci U S A 2007; 104: 13756-13761.

32. Tivol EA, Boyd SD, McKeon S, et al. CTLA4lg prevents lymphoproliferation and fatal multiorgan tissue destruction in CTLA-4-deficient mice. J Immunol 1997; 158: 5091-5094.

33. Qureshi OS, Zheng Y, Nakamura K, et al. Trans-endocytosis of CD80 and CD86: A molecular basis for the cell-extrinsic function of CTLA-4. Science 2011; 332: 600-603.

34. Qureshi O, Kaur S, Hou TZ, et al. Constitutive clathrin-mediated endocytosis of CTLA-4 persists during T cell activation. J Biol Chem 2012; 287: 9429-9440.

35. Onishi Y, Fehervari Z, Yamaguchi T, Sakaguchi S. Foxp3+ natural regulatory $T$ cells preferentially form aggregates on dendritic cells in vitro and actively inhibit their maturation. Proc Natl Acad Sci U S A 2008; 105: 10113-10118.

36. Oderup C, Cederbom L, Makowska A, Cilio CM, Ivars F. Cytotoxic $T$ lymphocyte antigen-4-dependent down-modulation of costimulatory molecules on dendritic cells in $\mathrm{CD} 4+\mathrm{CD} 25+$ regulatory T-cell-mediated suppression. Immunology 2006; 118: 240-249.

37. Kastenmuller W, Gasteiger G, Subramanian N, et al. Regulatory T cells selectively control CD8+ T cell effector pool size via IL-2 restriction. J Immunol 2011; 187: 3186-3197.

38. Riella LV, Sayegh MH. T-cell co-stimulatory blockade in transplantation: Two steps forward one step back. Expert Opin Biol Ther 2013; 13: 1557-1568.

39. Maltzman JS, Turka LA. T-cell costimulatory blockade in organ transplantation. Cold Spring Harb Perspect Med 2013; 3: a015537.

40. Genovese MC, Schiff $M$, Luggen $M$, et al. Longterm safety and efficacy of abatacept through 5 years of treatment in patients with rheumatoid arthritis and an inadequate response to tumor necrosis factor inhibitor therapy. J Rheumatol 2012; 39: 1546-1554.

41. Larsen CP, Knechtle SJ, Adams A, Pearson T, Kirk AD. A new look at blockade of T-cell costimulation: A therapeutic strategy for longterm maintenance immunosuppression. Am J Transplant 2006; 6: 876-883.

42. Larsen $\mathrm{CP}$, Pearson TC, Adams AB, et al. Rational development of LEA29Y (belatacept), a high-affinity variant of CTLA4-lg with potent immunosuppressive properties. Am J Transplant 2005; 5 : 443-453.

43. Vincenti F, Dritselis A, Kirkpatrick P. Belatacept. Nat Rev Drug Discov 2011; 10: 655-656. 
44. Wojciechowski D, Vincenti F. Challenges and opportunities in targeting the costimulation pathway in solid organ transplantation. Semin Immunol 2011; 23: 157-164.

45. Tao X, Constant S, Jorritsma P, Bottomly K. Strength of TCR signal determines the costimulatory requirements for Th1 and Th2 CD4+ T cell differentiation. J Immunol 1997; 159: 59565963.

46. Gogishvili T, Luhder F, Goebbels S, Beer-Hammer S, Pfeffer K, Hunig T. Cell-intrinsic and -extrinsic control of Treg-cell homeostasis and function revealed by induced CD28 deletion. Eur J Immunol 2013; 43: 188-193.

47. Tai $X$, Cowan $M$, Feigenbaum L, Singer A. CD28 costimulation of developing thymocytes induces Foxp3 expression and regulatory T cell differentiation independently of interleukin 2 . Nat Immunol 2005; 6: 152-162.

48. Zhang R, Huynh A, Whitcher G, Chang J, Maltzman JS, Turka LA. An obligate cell-intrinsic function for CD28 in Tregs. J Clin Invest 2013; 123: 580-593.

49. Gabrysova L, Christensen JR, Wu X, Kissenpfennig A, Malissen B, O'Garra A. Integrated T-cell receptor and costimulatory signals determine TGF-beta-dependent differentiation and maintenance of Foxp3+ regulatory T cells. Eur J Immunol 2011; 41: 12421248.

50. Semple K, Nguyen A, Yu Y, Wang H, Anasetti C, Yu XZ. Strong $\mathrm{CD} 28$ costimulation suppresses induction of regulatory $\mathrm{T}$ cells from naive precursors through Lck signaling. Blood 2011; 117: 3096-3103.

51. Riella LV, Liu T, Yang J, et al. Deleterious effect of CTLA4-Ig on a Treg-dependent transplant model. Am J Transplant 2012; 12: 846855.

52. Vincenti $F$, Charpentier $B$, Vanrenterghem $Y$, et al. A phase III study of belatacept-based immunosuppression regimens versus cyclosporine in renal transplant recipients (BENEFIT study). Am J Transplant 2010; 10: 535-546.

53. Vincenti $F$, Larsen $C$, Durrbach $A$, et al. Costimulation blockade with belatacept in renal transplantation. N Engl J Med 2005; 353: 770-781.

54. Poirier N, Blancho G, Vanhove B. CD28-specific immunomodulating antibodies: What can be learned from experimental models? Am J Transplant 2012; 12: 1682-1690.
55. Luhder F, Huang $Y$, Dennehy KM, et al. Topological requirements and signaling properties of T cell-activating, anti-CD28 antibody superagonists. J Exp Med 2003; 197: 955-966.

56. Kitazawa Y, Fujino M, Li XK, et al. Superagonist CD28 antibody preferentially expanded Foxp3-expressing nTreg cells and prevented graft-versus-host diseases. Cell Transplant 2009; 18 : 627-637.

57. Kitazawa Y, Fujino M, Sakai T, et al. Foxp3-expressing regulatory $T$ cells expanded with CD28 superagonist antibody can prevent rat cardiac allograft rejection. J Heart Lung Transplant 2008; 27: 362-371.

58. Chen J, Xie L, Toyama S, et al. The effects of Foxp3-expressing regulatory $T$ cells expanded with CD28 superagonist antibody in DSS-induced mice colitis. Int Immunopharmacol 2011; 11: 610617.

59. Hunig T. The storm has cleared: Lessons from the CD28 superagonist TGN1412 trial. Nat Rev Immunol 2012; 12: 317-318.

60. Tabares P, Berr S, Romer PS, et al. Human regulatory $T$ cells are selectively activated by low-dose application of the CD28 superagonist TGN1412/TAB08. Eur J Immunol 2014; 44: 1225-1236.

61. Vanhove B, Laflamme G, Coulon F, et al. Selective blockade of CD28 and not CTLA-4 with a single-chain Fv-alpha1-antitrypsin fusion antibody. Blood 2003; 102: 564-570.

62. Poirier N, Azimzadeh AM, Zhang T, et al. Inducing CTLA-4dependent immune regulation by selective CD28 blockade promotes regulatory $\mathrm{T}$ cells in organ transplantation. Sci Transl Med 2010; 2: 17ra0.

63. Poirier N, Mary C, Dilek N, et al. Preclinical efficacy and immunological safety of FR104, an antagonist anti-CD28 monovalent Fab' antibody. Am J Transplant 2012; 12: 2630-2640.

64. Zhang T, Fresnay S, Welty E, et al. Selective CD28 blockade attenuates acute and chronic rejection of murine cardiac allografts in a CTLA-4-dependent manner. Am J Transplant 2011; 11: 15991609.

65. Suchard SJ, Davis PM, Kansal S, et al. A monovalent anti-human CD28 domain antibody antagonist: Preclinical efficacy and safety. J Immunol 2013; 191: 4599-4610.

66. Liu D, Krummey SM, Badell IR, et al. 2B4 (CD244) induced by selective CD28 blockade functionally regulates allograft-specific CD8+ T cell responses. J Exp Med 2014; 211: 297-311. 